\title{
Not All Autoimmune Gastritis Are Created the Same
}

\author{
Massimo Rugge ${ }^{a}$, b, e, Robert M. Genta ${ }^{c}$, Ludovica Bricca ${ }^{\text {b, d }}$, \\ Edoardo Savarino $^{\mathrm{d}}$
}

\section{To the Editor}

We read with great interest the outstanding scientific paper recently published in Gastroenterology Research by Saulino et al, entitled "Characterization of Chronic Gastritis in Lynch Syndrome Patients with Gastric Adenocarcinoma" [1]. By concluding that "... most gastric adenocarcinoma in Lynch syndrome patients were located in the fundus and/or body ..., with a tumorigenesis of chronic gastritis (immune gastritis in most cases and Helicobacter pylori (H. pylori) gastritis in the minority of cases)-associated intestinal metaplasia, dysplasia, and carcinoma", the authors suggest gastric autoimmunity as electively involved in gastric oncogenesis.

Because of our long-standing interest in the still controversial assessment of gastric cancer (GC) risk associated with autoimmune gastritis, we would like to briefly comment on the results and the conclusions of the paper by Saulino et al [1-5].

The increased risk of GC in Lynch syndrome is well established. In their manuscript Saulino et al further support the hypothesis, previously put forward by Adar et al of a causative relationship between $\mathrm{MSH} 2$ mutation (as observed in mismatch repair-related Lynch syndrome) and autoimmune gastritis, and, as a consequence, between autoimmune gastritis and gastric adenocarcinoma [6-8].

In a retrospective series of 562 histologically consistent, serologically confirmed autoimmune gastritis, we detected neoplastic lesions (both non-invasive and invasive) in only four high-stage gastritis patients (Operative Link on Gastritis Assessment (OLGA) stages III and IV), three of whom with a history of $H$. pylori infection [2, 7]. In a second long-term prospective follow-up study (data on file) of 258 patients with autoimmune gastritis who tested negative for $H$. pylori infection

Manuscript submitted June 29, 2021, accepted July 26, 2021

Published online November 5, 2021

aDepartment of Medicine (DIMED), Surgical Pathology \& Cytopathology Unit, Padua University, Padua, Italy

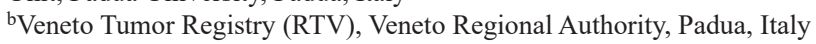
'Department of Pathology, Michael E. DeBakey VA Medical Center, Baylor College of Medicine, Houston, TX, USA

${ }^{\mathrm{d} D e p a r t m e n t}$ of Surgical Oncological \& Gastroenterological Sciences (DiSCOG), Gastroenterology Unit, Padua University, Padua, Italy

${ }^{\mathrm{e}}$ Corresponding Author: Massimo Rugge, Department of Medicine (DIMED), Surgical Pathology \& Cytopathology Unit, Padova University, Padua, Italy. Email: massimo.rugge@unipd.it
(H. pylori serology and molecular testing on gastric biopsy tissue specimens) and assessed by paired endoscopy/histology, two cases of low-grade gastric dysplasia were histologically assessed, invasive gastric epithelial malignancies were never documented, and only two cancer cases (one breast, and one lung) emerged during a mean follow-up time of 7 years (range: 2 - 17 years). Neither of these figures supports an increased risk of GC in patients with autoimmune gastritis, particularly when an etiological involvement of $H$. pylori is excluded.

In keeping with previous findings by Adar et al [6], the cohort of GC patients described by Saulino refers to a very peculiar biological-clinical setting, characterized by the combination of a cancer-promoting genetic profile (MSH2/MLH1 abnormality) with an autoimmune-based gastritis [1].

The loss of function/mutation of MSH2 (one of the most involved genetic defects resulting into Lynch syndrome) has been consistently included among the features associated with increased tumor mutational burden (TMB), which is in turn involved in T-cell response against tumor cells [4, 8-10]. Such a biological setting evokes the hypothesis of a common "syndromic" background (mismatch repair genes/MMR deficiency) for both Lynch syndrome and autoimmune diseases [11, 12]. Such an intriguing theory, however, cannot, per se, support linking the entire spectrum of autoimmune gastritis to MMR, ultimately associating (longstanding) gastric autoimmunity to a significantly increased risk of GC.

Among the 11 patients included in the Saulino's study (after excluding three cases with reactive gastropathy of normal antrum), five featured the inflammatory/atrophic involvement of the antral mucosa, and no information is provided on the antral mucosa of the three remaining patients [1]. While these features do not exclude a potential role for gastric autoimmunity in the onset of GC, they leave still open the question of the role of $H$. pylori infection (active, previous, or undetected) as co-responsible for the cancerization field in which GC mostly develops.

In conclusion, the results obtained by Saulino et al stimulate interesting speculations on the biological relationships between Lynch syndrome, a subset of autoimmune gastritis, and non-gastric autoimmune diseases. These results, however, should be interpreted carefully before suggesting that non-Lynch-associated (i.e., "non-syndromic") autoimmune gastritis could be a significant determinant in increasing the risk for GC.

\section{Acknowledgments}

None to declare. 


\section{Financial Disclosure}

Authors have no financial disclosure to report.

\section{Conflict of Interest}

The authors declare that they have no conflict of interest concerning this article.

\section{Informed Consent}

Not applicable.

\section{Author Contributions}

Massimo Rugge and Robert M. Genta: critical evaluation of the manuscript to which the Letter to the Editor is addressed, and drafting the manuscript and approval of the final version of the submitted manuscript. Ludovica Bricca: critical evaluation of the available literature on the addressed topic, and drafting of the manuscript and approval of the final version of the manuscript. Edoardo Savarino: critical evaluation and approval of the final version of the submitted manuscript.

\section{Data Availability}

The authors declare that data supporting the findings of this study are available within the article.

\section{References}

1. Saulino D, Chen R, Wang K, Shen M, Zhang X, Westerhoff $\mathrm{M}$, Cheng J, et al. Characterization of chronic gastritis in lynch syndrome patients with gastric adenocarci- noma. Gastroenterology Res. 2021;14(1):13-20.

2. Rugge M, Fassan M, Pizzi M, Zorzetto V, Maddalo G, Realdon S, De Bernard M, et al. Autoimmune gastritis: histology phenotype and OLGA staging. Aliment Pharmacol Ther. 2012;35(12):1460-1466.

3. Song M, Latorre G, Ivanovic-Zuvic D, Camargo MC, Rabkin CS. Autoimmune diseases and gastric cancer risk: a systematic review and meta-analysis. Cancer Res Treat. 2019;51(3):841-850.

4. Lenti MV, Rugge M, Lahner E, Miceli E, Toh BH, Genta $\mathrm{RM}$, De Block C, et al. Autoimmune gastritis. Nat Rev Dis Primers. 2020;6(1):56.

5. Rugge M. Gastric cancer risk: between genetics and lifestyle. Lancet Oncol. 2020;21(10):1258-1260.

6. Adar T, Friedman M, Rodgers LH, Shannon KM, Zukerberg LR, Chung DC. Gastric cancer in Lynch syndrome is associated with underlying immune gastritis. J Med Genet. 2019;56(12):844-845.

7. Rugge M, Savarino E, Sbaraglia M, Bricca L, Malfertheiner P. Gastritis: The clinico-pathological spectrum. Dig Liver Dis. 2021;53(10):1237-1246.

8. Park DI, Park SH, Kim SH, Kim JW, Cho YK, Kim HJ, Sohn CI, et al. Effect of Helicobacter pylori infection on the expression of DNA mismatch repair protein. Helicobacter. 2005;10(3):179-184.

9. Jia M, Yao L, Yang Q, Chi T. Association of MSH2 expression with tumor mutational burden and the immune microenvironment in lung adenocarcinoma. Front Oncol. 2020;10:168.

10. Tamura K, Kaneda M, Futagawa M, Takeshita M, Kim S, Nakama M, Kawashita N, et al. Genetic and genomic basis of the mismatch repair system involved in Lynch syndrome. Int J Clin Oncol. 2019;24(9):999-1011.

11. Muro Y, Sugiura K, Mimori T, Akiyama M. DNA mismatch repair enzymes: genetic defects and autoimmunity. Clin Chim Acta. 2015;442:102-109.

12. Toledano H, Orenstein N, Sofrin E, Ruhrman-Shahar N, Amarilyo G, Basel-Salmon L, Shuldiner AR, et al. Paediatric systemic lupus erythematosus as a manifestation of constitutional mismatch repair deficiency. J Med Genet. 2020;57(7):505-508. 